\title{
Flat Optical Fiber Daylighting System with Lateral Displacement Sun-Tracking Mechanism for Indoor Lighting
}

\author{
Ngoc Hai Vu and Seoyong Shin * \\ Department of Information and Communication Engineering, Myongji University, 116 Myongji-ro, Cheoin-gu, \\ Yongin, Gyeonggi-do 17058, Korea; anh_haicntn@yahoo.com \\ * Correspondence: sshin@mju.ac.kr; Tel.: +82-102-709-6483
}

Academic Editor: Hongxing Yang

Received: 28 September 2017; Accepted: 20 October 2017; Published: 23 October 2017

\begin{abstract}
An essential impact which can improve the indoor environment and save on power consumption for artificial lighting is utilization of daylight. Optical fiber daylighting technology offers a way to use direct daylight for remote spaces in a building. However, the existing paradigm based on the precise orientation of sunlight concentrator toward the Sun is very costly and difficult to install on the roof of buildings. Here, we explore an alternative approach using mirror-coated lens array and planar waveguide to develop a flat optical fiber daylighting system (optical fiber daylighting panel) with lateral displacement Sun-tracking mechanism. Sunlight collected and reflected by each mirror-coated lens in a rectangular lens array is coupled into a planar waveguide using cone prisms placed at each lens focus. This geometry yields a thin, flat profile for Sunlight concentrator. Our proposed concentrating panel can be achieved with $35 \mathrm{~mm}$ thickness while the concentrator's width and length are $500 \mathrm{~mm} \times 500 \mathrm{~mm}$. The commercial optical simulation tool (LightTools ${ }^{\mathrm{TM}}$ ) was used to develop the simulation models and analyze the system performance. Simulation results based on the designed system demonstrated an optical efficiency of $51.4 \%$ at a concentration ratio of 125. The system can support utilizing a lateral displacement Sun-tracking system, which allows for replacing bulky and robust conventional rotational Sun-tracking systems. This study shows a feasibility of a compact and inexpensive optical fiber daylighting system to be installed on the roof of buildings.
\end{abstract}

Keywords: optical fiber daylighting system; planar waveguide; plastic optical fiber; solar energy; concentrator

\section{Introduction}

The Sun is the most plentiful energy source for the Earth, and is a renewable, sustainable and totally inexhaustible energy source. One of the direct applications of solar energy is daylighting. Daylight is used to illuminate building interiors to affect the indoor environment, health, lighting quality, and energy efficiency [1,2]. In sustainable buildings, daylighting can provide energy reductions through the use of electric light controls, and it can reduce the dependence on artificial lighting, which cannot fulfill the needs of the human body. Krarti M. et al. have estimated that the energy consumption for artificial lighting in the building can be reduced $50 \%$ to $80 \%$ by efficient utilization of daylighting [3]. However, the high cost and low optical efficiency are challenges of any daylighting system. Recently, many research groups have been concentrating on these problems by simplifying a daylighting system design as a cost-effective solution. In terms of delivery of natural light to remote areas in a building where daylight is limited, the most efficient solution is optical fiber-based daylighting technology. A typical fiber optic lighting system consists of a sunlight concentrator mounted with a Sun-tracking 
system, optical fiber cables, and light diffuser [4]. The optical fiber daylighting systems have been developed for the past 20 years [5], and many studies have been published in scientific journals [5-8]. A few of them were commercialized in the market such as Parans Solar Lighting systems (Gothenburg, Sweden), Himawari system (Tokyo, Japan), etc. [9,10].

In conventional optical fiber daylighting systems (OFDS), the concentrators employ two different mechanisms: a refractive mechanism such as a convex lens or Fresnel lens, or a reflective mechanism such as a parabolic mirror [11]. One end of the plastic optical fiber is aligned at the focal point of the concentrator. The optical fiber bundle transports light into the interior to be illuminated, and the fiber optic luminaires (i.e., bundles of fiber ends) launch the light into the room. Concentrated sunlight is coupled into optical fiber bundle and guided to interior. A fiber optic luminaire is attached to other fiber ends to distribute the light into the room [12]. Many studies in this technology have been reported; however, the commercialization process is delayed due to technical problems. Fresnel lenses or parabolic mirrors usually have long focal lengths, making OFDS heavy and cumbersome. Furthermore, Sun-tracking systems are required to precisely rotate the concentration system to follow with the sunlight direction. Because every OFDS is working outdoors, Sun-tracking systems usually require a bulky pedestal, and they must be mechanically stable against wind-loading forces [13-15]. The bulk and robustness issues of concentrator and Sun-tracking systems make the OFDS become costly and inhibit the use of them on the roof of the building.

In order to reduce the complexity of existing OFDSs, an alternative approach for the sunlight concentrator is proposed by using a flat solar concentrator with lateral displacement Sun-tracking mechanism based on the utilization of mirror-coated lens array and planar waveguide. The original concept of a solar concentrator based on a planar waveguide came from a design by R. Winston et al. [16] and backlight-reversed concentration optics by M. Chen et al. [17]. Some types of planar waveguide concentrators have been proposed in [18-22] with applications in concentrated photovoltaic (CPV). Figure 1a shows the principles of a planar waveguide concentrator. In this case, the sunlight, which is collected and concentrated by an array of lenses (plano-convex lenses), was coupled into a planar waveguide by the prisms integrated on the surface of the waveguide. Rays that satisfy the total internal reflection (TIR) condition propagate through the waveguide to the exit aperture, typically at the edge of the slab. Some of the sunlight rays leak out of waveguide as decoupling loss because they strike and decouple at subsequent directing surfaces (prism). The thickness of concentrator is determined by the focal length of the lens. Assume that the curvature radius of each plano-convex lens is $R$, so its focal length is calculated by a simple equation representing a plano-convex lens $f=R /(n-1) \cong 2 R$, where, $n \cong 1.5$ is the refractive index of the lens material. The focal length of each lenslet is comparatively long relative to its diameter. As shown in Figure 1a, in a larger-scale concentrator using an array of large-size lenses, the system becomes very thick. In addition, the system still requires a sophisticated rotational Sun-tracking system to follow the Sun position. Here, we propose a novel OFDS using a lens array with mirror coating at the curvature surface. Figure $1 \mathrm{~b}$ shows ray-tracing analysis of a mirror-coated lens array. In this specialized design, each lens concentrates sunlight by a reflection mechanism rather than by refraction in usual designs. With mirror coating on the curvature surface, each lens becomes a concave mirror whose focal length, $f$, is half of the curvature radius, $R$. This reduces the focal length to a quarter, in this case providing the basis for designing a flat OFDS. Similar to conventional planar waveguide concentrator, our proposed design also utilizes the planar waveguide with prism etched at top surface to redirect and guide concentrated sunlight to the exit port. As shown in the lower and right part of Figure $1 \mathrm{~b}$ when the sunlight direction changes, the focal point of each lens moves along a straight line. A lateral movable waveguide can track the position of focal points and align the prism at the center of them. This makes it possible for a very simple flat solar concentration system with lateral displacement Sun-tracking mechanism to replace the conventional design, which requires a very bulky concentrator and a robust Sun-tracking system based on a rotational mechanism. In this study, we propose a novel approach based on using two-dimensional (2D) array of mirror-coated spherical lenses and planar waveguide instead of the 
conventional lens array. This approach can achieve higher optical efficiency and allows replacing rotational Sun-tracking to lateral displacement Sun-tracking.

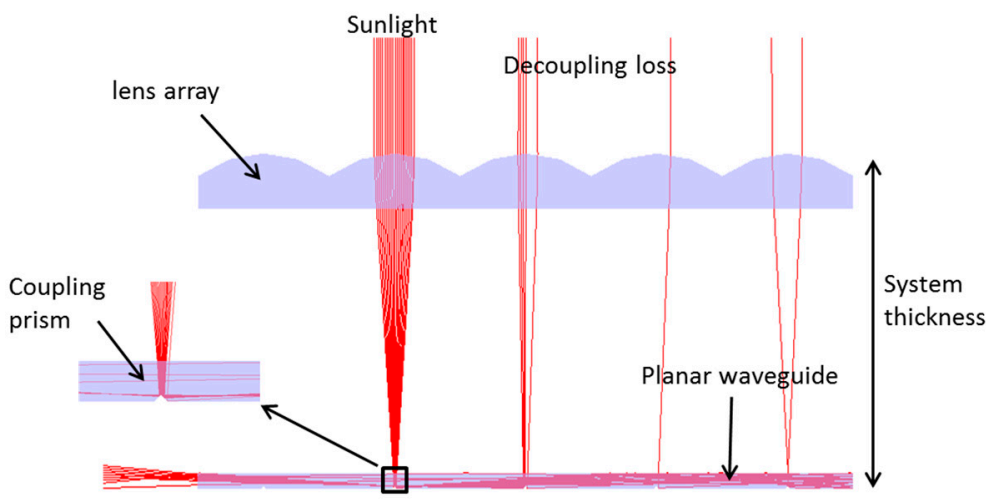

(a)

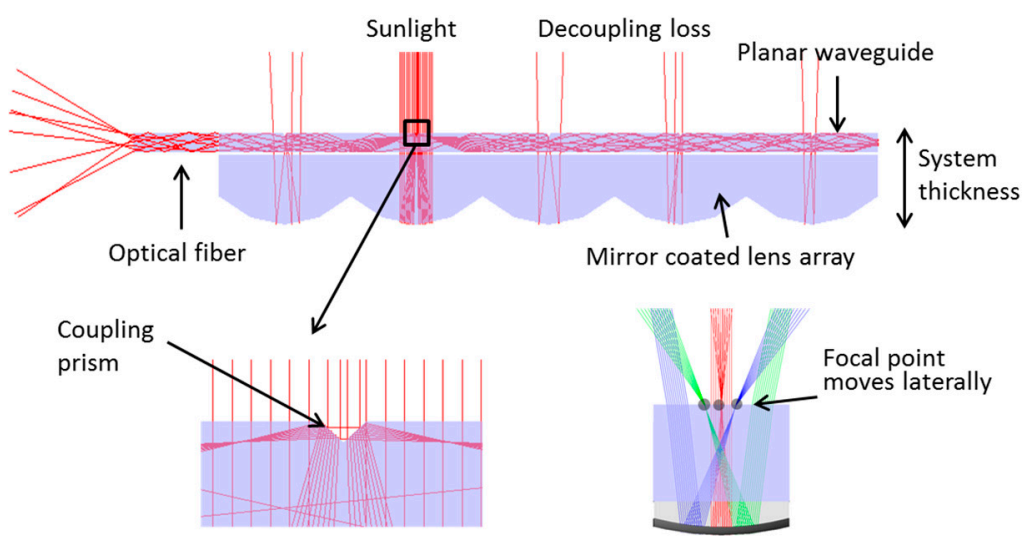

(b)

Figure 1. (a) Principles of a conventional planar waveguide concentrator; (b) Our proposed flat optical fiber daylighting systems (OFDS) which allows using lateral displacement Sun-tracking mechanism.

The next parts of the paper will discuss details on the system design and performance. We describe a proposed design of a OFDS using 2D spherical lenses array. In Section 3, a flat OFDS based on 2D array of lenses is modeled in LightTools ${ }^{\mathrm{TM}}$ software to analyse the optical efficiency and Sun-tracking performance of such systems. Section 4 is the final conclusion, including the remarks and possibilities for future works.

\section{Design of Flat OFDS}

An efficient OFDS must capture sunlight through a concentrator and then focus it on a small area with high efficiency and low fabrication cost. In the literature, many techniques have been proposed to collect sunlight by reflectors or lenses. However, these designs require the bulky and robustly rotational Sun-tracking systems, which occupy a large part of initial and maintenance cost.

The design for a flat OFDS using mirror-coated lens array and planar waveguide proposed in this study can overcome these challenges. The flow chart of the system is shown in Figure 2. The proposed sunlight concentrator part is composed of lens arrays with a mirror coating and planar waveguide. The planar waveguide, which is placed on top of the lens array, consists of a transparent sheet integrated with prisms etched at the top surface. The lens array is fixed, and the planar waveguide can slide freely between the lens arrays. The gap between lens array and waveguide is lubricated by transparent fluid that is also index-matched to reduce Fresnel reflection losses at the gap region. 
The waveguide is mounted to a lateral displacement Sun-tracking system. The role, optical mechanism and design parameters of each component in the proposed OFDS are discussed in Sections 2.1-2.3.

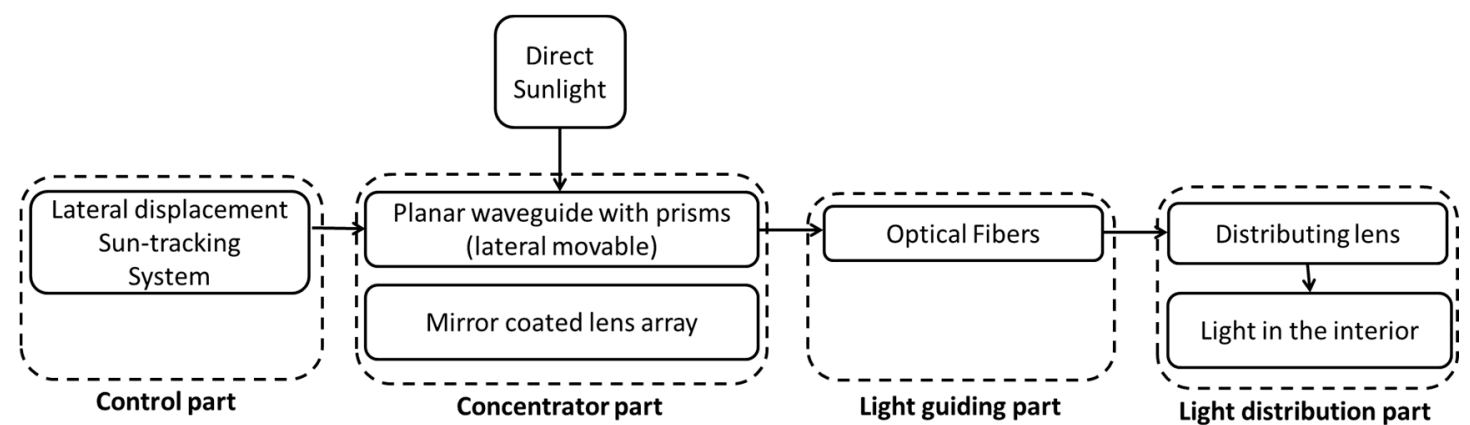

Figure 2. Flow chart of the proposed flat OFDS.

\subsection{Array of Spherical Lenses}

In this subsection, the sunlight concentrator is constructed from a rectangular array of poly-methyl methacrylate (PMMA) spherical lenses as shown in Figure 3. Some main parameters of the lens array are also depicted in Figure 3, namely: the size of lenslet array $L$, size of a single lenslet $d$, radius of curvature of lens $r$, and the thickness of the lenslet array (or focal length) $f$.

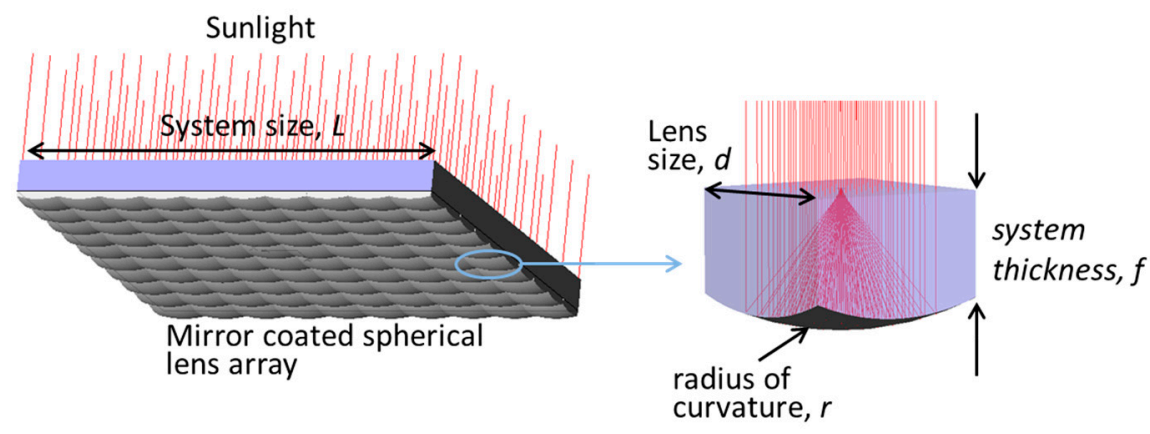

Figure 3. A mirror-coated spherical lens array and its associated parameter and Ray-tracing analysis of a singlet lens.

In our proposed design, 100 lenses of $d=50 \mathrm{~mm}$ and curvature radius $r=75 \mathrm{~mm}$ are arranged in a square array as shown in Figure 4a so that the total length of the system is $L=500 \mathrm{~mm}$ and thickness $f=35 \mathrm{~mm}$. The size of the lens array can be chosen freely depending on each specific case. However, increasing the size of lens $\mathrm{d}$ and curvature radius $r$ will increase the thickness of the system, whereas reducing the lens size increases the number of lenses and it makes the system more complicated. Using parameter values of commercially available lens array for the system design is a good solution to reduce system cost. In our proposed design, we utilized the parameters of commercial fly eye lens array from the company OptoSigma. This lens array is being used for making illumination light of homogeneous brightness for projectors. For the purpose of designing a flat OFDS for rooftop installation, lens size $d=50 \mathrm{~mm}$ and $r=75 \mathrm{~mm}$ are reasonable because the thickness of the system should be limited to $35 \mathrm{~mm}$, which is similar to the thickness of a typical commercial photovoltaic module for rooftop application. The focused regions of the lens array are one hundred points distributed in a 2D array on the focal plane. Figure 4a shows the concentrated light on the focal plane when the sunlight is perpendicular to lens array surface. When the sunlight deviated with an incident angle of $\theta$, it is dispersed at the flat surface of the lens array. Then the focal area of each lens will be broadened. In order to quantify the effect of sunlight deviation, $\theta$ on the distribution of sunlight on the focal plane, the ray-tracing analysis was conducted by varying the sunlight direction. The 
focused area is a design parameter for the planar waveguide and light-directing prism. Simulations were performed with sunlight deviations of $\theta=0^{\circ}$ to $70^{\circ}$. Figure $4 \mathrm{~b}$ shows the variation of the size of a focal point with the sunlight incident angle (note that the dimensions of the focal region are locally exaggerated for convenient explanation).

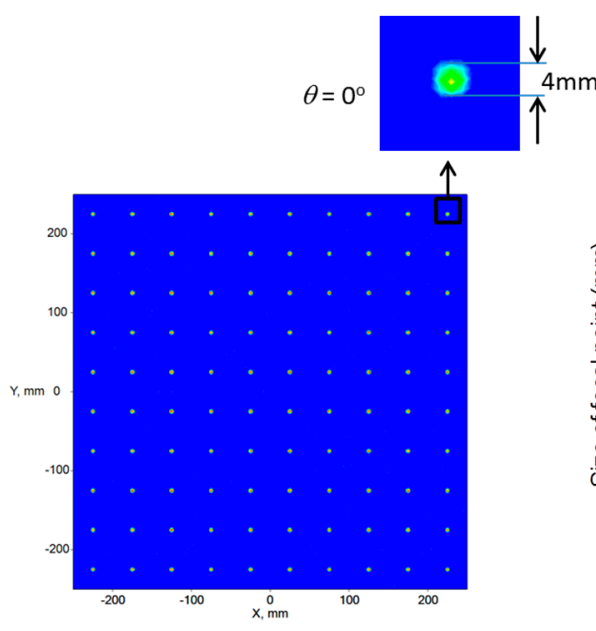

(a)

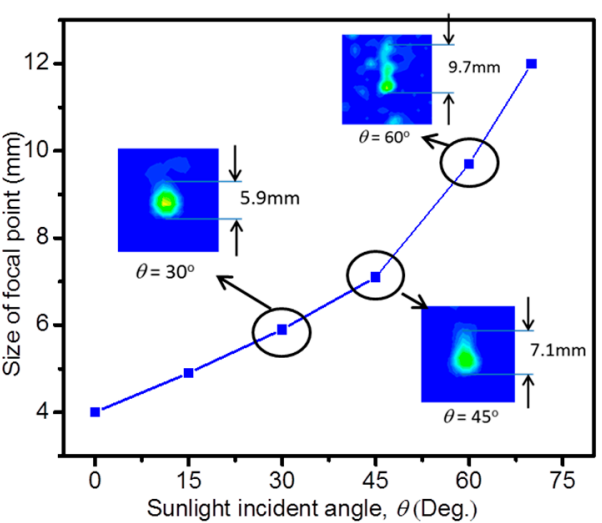

(b)

Figure 4. (a) Distribution of concentrated sunlight on the focal plane of mirror-coated lens array; (b) the dependence of focal area on the sunlight incident angle.

\subsection{Planar Waveguide}

The role of the planar waveguide is coupling and guiding the light which is concentrated by mirror-coated lens array to its exit port. As shown in Figure 2, this part lies on the top of the mirror-coated lens array. The sunlight passes through the planar waveguide then reflects back to the waveguide by the mirror-coated lens array and is coupled into waveguide using mirror-coated prisms positioned at each lens focus. The prisms reflect light onto a planar waveguide. The structure of prism is calculated so that reflected rays satisfy the TIR condition of waveguide. The exit port is one edge of the waveguide which works as a transmitting surface. Three other edges are coated mirror, so the sunlight that reaches to these edges will reflect back to the exit port. The detail of planar waveguide with the light-directing prism is shown in Figure $5 \mathrm{a}$. Figure $5 \mathrm{~b}$ shows a cross-section of a flat concentrator based on mirror-coated spherical lens array and planar waveguide with ray-tracing analysis. It is clear to see that sunlight passes through planar waveguide and reflects back by mirror-coated lenses and then it is redirected by prisms and escapes to exit port by TIR phenomenon. There are few rays which escape at the surface of waveguide due to decoupling with prisms (decoupling loss).

As shown in Figure 5a, the prims have a cone shape with a mirror coating. In order to accommodate all of the sunlight input, the cone diameter must be larger than that of the focal area size. Although increasing the prism size will widen the acceptance angle of sunlight direction, it will also increase the decoupling loss of the system. In this situation, the appropriate cone prism diameter is $7.1 \mathrm{~mm}$ corresponding to a sunlight acceptance angle of $45^{\circ}$. The thickness of waveguide should be larger than the height of the cone prism $(3.55 \mathrm{~mm})$. Each lens element in the lens array has its own geometric concentration $C_{\text {lens }}$ defined by the ratio between lens area and cone prism bottom area. The loss mechanism inside the planar waveguide which has relation with the $C_{\text {lens }}$ will be detailed in the next Section. An important property of the OFDS, the geometric concentration ratio, $C_{\text {geo }}$, is given by:

$$
C_{\text {Geo }}=\frac{\text { waveguide length }}{\text { waveguide thickness }}=\frac{L}{w}
$$


Increasing the length $(L)$ of concentration system can increase the concentration ratio. However, there is a trade-off between the concentration ratio and optical efficiency. The analytic model of the planar concentrator with spherical lenses which explain the relation between optical efficiency and the concentration ratio has already been presented in previous papers $[20,23]$. When the sunlight propagates and transports to the exit by planar waveguide, some of the rays hit the redirecting prism which was integrated on the waveguide surface, then they are decoupled from the waveguide. This is loss mechanism of planar waveguide concentrator. In order to perform a qualitative analysis of the relation between optical efficiency and waveguide parameters, a brief synthesis about the theoretical calculation of flat concentrator based on the use of mirror-coated lens array for coupling of light in the waveguide is presented here. The theoretical calculation for optical efficiency of waveguide concentrator was defined by Karp by following the equations in [23],

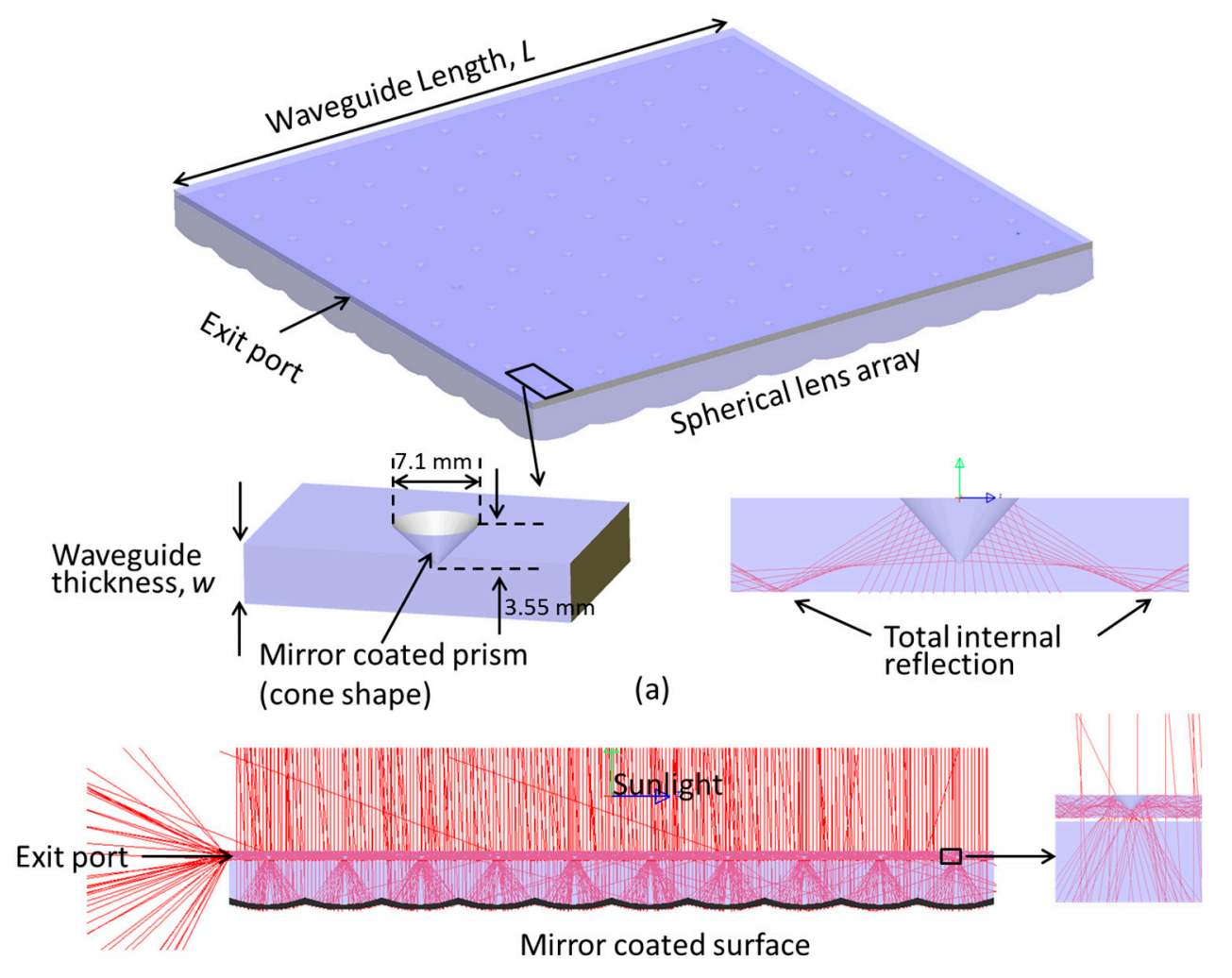

(b)

Figure 5. (a) A planar waveguide with mirror-coated prisms placed on the top of the lens array. A prism is exaggerated for convenient interpretation and ray-tracing analysis is applied to illustrate how the sunlight decouples inside the waveguide; (b) Ray tracing on a planar waveguide combined with a mirror-coated spherical lens array to illustrate planar waveguide collects light from different lenses and transports it to the exit port.

$$
\begin{gathered}
\eta_{\text {decouple }}(P, \varphi)=\left(1-\frac{1}{C_{\text {lens }}}\right)^{\frac{P \tan \varphi}{22 w}} \\
\eta_{\text {position }}(P, \varphi)=R \times \eta_{\text {decouple }} \times \exp (-\alpha P / \cos \varphi) \\
\eta_{\text {total }}=\frac{\sum_{P} \int_{0}^{\varphi_{\max }} \eta_{\text {postion }}(P, \varphi)}{(L-r) / 2 r} ; P=r, 3 r, 5 r, L-r / 2 r
\end{gathered}
$$

where $C_{\text {lens }}$ is the concentration ratio of the mirror-coated lens, which is the ratio of lens size and focal point size. In this study, the $C_{\text {lens }}$ was discussed and determined in Section 2.2; $w$ is the waveguide 
thickness which was indicated in Figures 5a and 6; L is the waveguide length; $\mathrm{P}$ is the position of prism which is the distance to the exit surface and $2 r$ (or $d$, lens size) is the pitch of the lens array which was indicated in Figures 3 and 6. Equation (2) calculates the optical efficiency for the light which is redirected at prism position $P$ with coupled angle $\varphi$ (the angle of the ray which is redirected by prism $P$ ). The parameters used in the calculation are associated with system structure as shown in Figure 6. In Equation (3), the exponential material attenuation $(\alpha)$ along the optical path and the reflective coefficient of the coupling prism, $R$, is taken into account. Equation (4) is an integration over all prism position $P$ and coupled angle to calculate total optical efficiency of a planar waveguide concentrator. As shown in Equations (1) and (4), it is clear that there is a trade-off between the optical efficiency and concentration ratio. When the light travels in the waveguide, the loss by material attenuation and decoupling becomes significant in case of scaling up the system. The dependence of optical efficiency on the some parameters of waveguide will be calculated by simulation in the next Section.

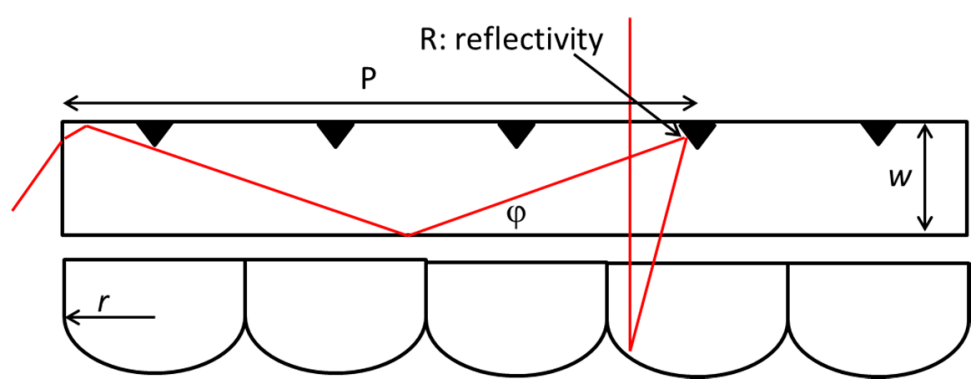

Figure 6. Lateral view of the waveguide with single ray-tracing to illustrate the theoretical calculation of optical efficiency.

\subsection{Plastic Optical Fiber Coupling}

Sunlight output from the exit port of the waveguide needs to deliver to the interior. For easy installation in the building, the most significant requirement of sunlight transportation methods is wiring feature. Therefore, only optical fibers, which can be installed as simply electrical wiring, are capable of fulfilling the requirement. In the daylighting application, the light transmission properties of optical fiber should be different from conventional ones in communication application. The fiber is required to transmit the visible range of sunlight with low attenuation loss. Although optical fiber based on silica have lower attenuation loss than plastic optical fiber (POF), POFs are widely used in daylighting systems because they are lower in production cost, more flexible for complex wiring in building. In our proposed system, typical POFs with a $2 \mathrm{~mm}$ core diameter which are designed for illumination purpose by Sichuan Huiyuan Plastic Optical Fiber Co., Ltd. (Chengdu, China), were utilized [24]. The fiber parameters are listed in Table 1.

Table 1. Plastic optical fiber (POF) parameters for design and simulation.

\begin{tabular}{cc}
\hline \multicolumn{2}{c}{ POF Technical Specifications } \\
\hline Attenuation & $0.46 \mathrm{~dB} / \mathrm{m}$ \\
Core/Cladding Diameter & $1.98 / 2.0$ \\
Refractive Index: Core/Cladding & $1.492 / 1.402$ \\
Minimum Bend Radius & $50 \mathrm{~mm}$ \\
Spectral Trans. Range & $380-750 \mathrm{~nm}$ \\
\hline
\end{tabular}

The size of exit port from planar waveguide is $4 \mathrm{~mm} \times 500 \mathrm{~mm}$ (Figure $4 \mathrm{a}$ ) and the optical fiber diameter is $2 \mathrm{~mm}$, so we proposed a $2 \times 250$ rectangular array of POFs bundle for appropriate coupling with waveguide as shown in Figure 7. The geometric coupling efficiency is calculated by the ratio of 
total area of fiber ends and exit port area, $\eta_{\text {fiber-coupling }}=0.75$. Multiple optical fibers were connected to the waveguide by polishing the ends of bundled fibers and simply putting the polished ends to the waveguide as tight as possible. The planar waveguide/POFs coupling loss consists of imperfection of the waveguide and POF endfaces, numerical aperture mismatch and Fresnel losses. We propose using the index matching gel between the air gap of POF bundle and waveguide to reduce Fresnel losses. The numerical aperture mismatching, which is a different angle between output beam from waveguide and acceptance angle of POF, also is a significant loss. Equation (5) shows the calculation of numerical angle of POFs.

$$
n_{0} \sin \theta=\sqrt{n_{c o}^{2}-n_{c l}^{2}}
$$

where $n_{c o}$ and $n_{c l}$ are the refractive index of the POF's core and cladding, respectively; $n_{0}$ is the refractive index of matching gel. Using the POF's parameter in Table 1, the acceptance angle of POF, $\theta= \pm 19^{\circ}$ was calculated. The angle of light beam that outputs from the exit port of waveguide can be estimated based on using Equation (5) with a cladding index of 1 (air) and a core index of 1.49 (index of PMMA). The angle of sunlight rays output from waveguide exit port is $\pm 49^{\circ}$. The big difference of POF acceptance angle and waveguide output beam angle shows that the numerical aperture mismatching loss is significant. The optical loss of POF coupling will be discussed based on simulation in Section 3 .

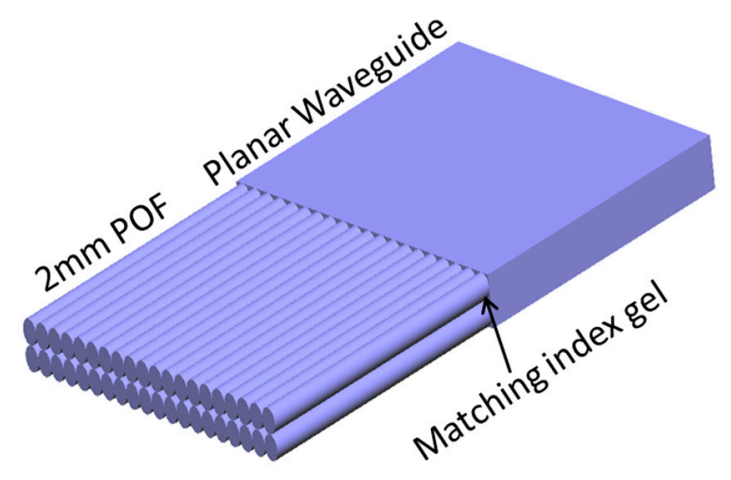

Figure 7. A ribbon arrangement of optical fiber is coupled with planar waveguide.

\section{Simulation Results and Discussion}

Commercial optical modeling software, LightTools ${ }^{\mathrm{TM}}$, was used to model and analyse the performance of proposed flat OFDS. The complete Sun geometry model, including the Sun spectrum supported by LightTools software, provides an easy method to evaluate the lateral displacement Sun-tracking possibility and the losses analysis of the system. In the designed system, we propose using one of the most common optical plastics, poly-methyl methacrylate (PMMA) with a refractive index of $n_{P M M A}=1.49$ for mirror-coated spherical lens array, planar waveguide, and optical fiber. We assume that the system is aligned along the north-south (NS) and east-west (EW) directions, as shown in Figure 8. The site of application was located at 127.0 longitude and 37.50 latitude. The concentrator surface is perpendicular to sunlight direction when Sun is at the highest solar elevation (zenith) angle of $76^{\circ}$ and takes place at 12:30 p.m. 


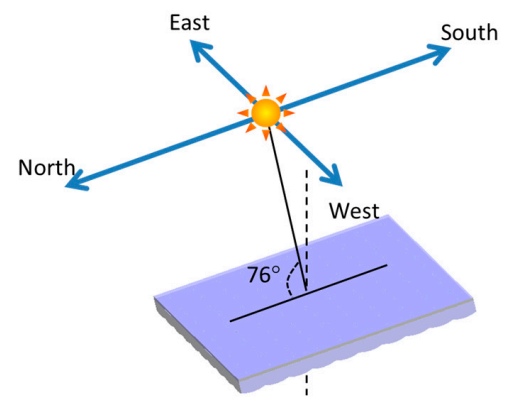

(a)

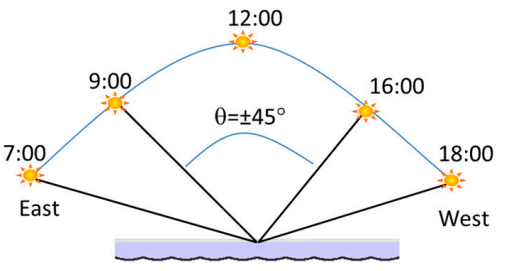

(b)

Figure 8. (a) Alignment of the system along the N-S and E-W directions; (b) Sun position at the different time of a day.

\subsection{Optical Efficiency of System}

Firstly, we evaluated the dependence of system efficiency on the concentration ratio $\mathrm{C}_{\text {geo. }}$. Because the length of concentrator was fixed at $L=500$, the concentration ratio will change with the variation of waveguide thickness. Simulation was carried with several different concentration ratio $C_{g e o}=71 \times, 83 \times, 100 \times$ and $125 \times$, corresponding with the waveguide thickness $w=7,6,5$ and $4 \mathrm{~mm}$, respectively. The concentrator optical efficiency was measured by the ratio between the total luminous flux at the surface of the waveguide and at the exit port. The simulation result is illustrated in Figure 9. The Fresnel losses, decoupling loss, material absorption, reflection loss at the surface of lenses and prisms were taken into account in the simulation. The decrease of optical efficiency when the concentration ratio increases, as shown in Figure 9, was predicted at qualitative analysis based on the theoretical model. The concentration ratio considerably affects the OFDS cost because a larger exit port will require a large number of POFs. The thickness of planar waveguide must be thicker than the height of the prism, which was $3.55 \mathrm{~mm}$ (Figure 5). In this design, the thickness waveguide $w=4 \mathrm{~mm}$ is reasonable. It corresponds with the concentration ratio of $125 \times$ and an optical efficiency of $76.3 \%$. To evaluate the losses of optical fiber coupling, a POF bundle was attached to the exit port and the luminous flux was measured at another end of the POF bundle. The optical efficiency is reduced to $51.4 \%$. The cause of this reduction is the non-matching between optical fiber bundle/exit port shape and difference of numerical aperture of $\mathrm{POF} /$ planar waveguide.

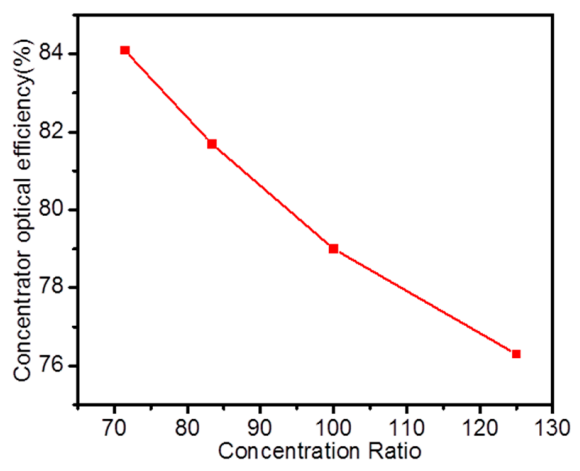

Figure 9. The dependence of concentrator optical efficiency and concentration ratio.

To evaluate the performance of our proposed OFDS and Sun-tracking mechanism, the output illuminance and the optical efficiency were calculated at different times of a summer day. The variation of optical efficiency and luminous flux (output at the end of POF) at different hours are calculated and shown in Figure 10a. One interesting observation from Figure 10 is that the optical efficiency was constant to $51.4 \%$ (including POF coupling loss) from 8:00 a.m. to 4:00 p.m.; it increased fast from 6:00 a.m. to 8:00 a.m. and decreased particularly fast at the time from 4:00 p.m. to 7:00 p.m. This can 
be explained by referring back to the design of coupling prisms. The size of prism was designed to cover sunlight focused area with the sunlight incident angle of $45^{\circ}$. When the sunlight incident angle is out of range of $\pm 45^{\circ}$ at 6:00 a.m.-8:00 a.m. and 4:00 p.m.-7:00 p.m., the prism size becomes smaller than focal area, so the efficiency drops so fast. The luminous flux $(\mathrm{lm})$ in the exit port at different times of the day is shown in Figure 10b. Losses due to Fresnel reflection, mirror reflection, optical fiber coupling, sun tracking, planar waveguide material absorption, and light distributors were included in this calculation.

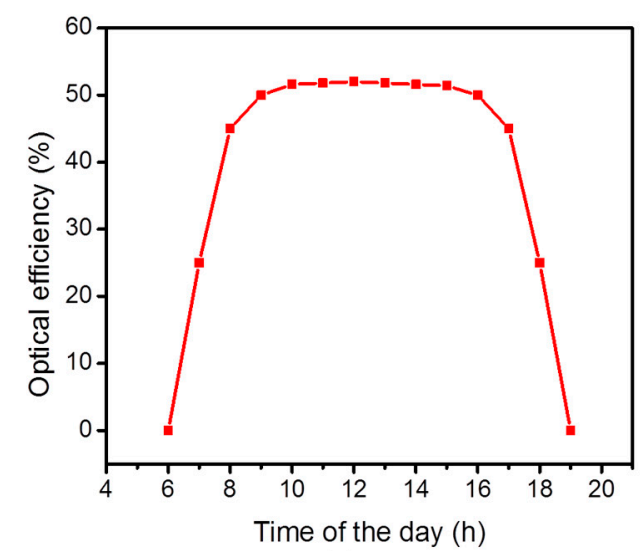

(a)

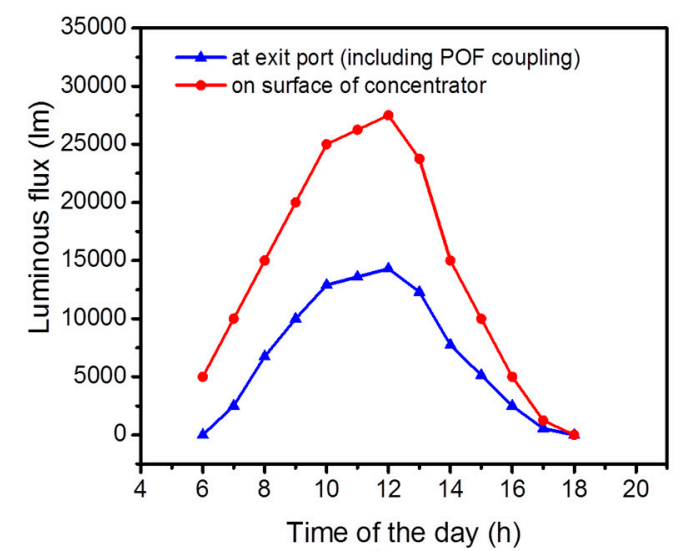

(b)

Figure 10. Flat OFDS performance over the course of the day including POF coupling loss. (a) The optical efficiency and output luminous flux changes depending on the position of the sun in the sky; (b) The dependence of luminous flux on the surface of the sunlight concentrator and the output at the exit port at different times during a sunny day.

\subsection{Lateral Displacement Sun-Tracking Performance}

For proper operation of the proposed flat OFDS, the sunlight should always be focused on the center of prisms; therefore, total focused sunlight beam is coupled into the waveguide. As discussed above, Sun-tracking for the proposed flat CPV system can be implemented by lateral movement of the waveguide with respect to the lens array layer. The moving part of the system (waveguide) is mounted on lens array layer with a two-axis lateral movement tracking system. To determine the amount of translation, we examined the focal point positions over a day. The focal point position $\delta$ (corresponds with the waveguide position) is defined as shown in Figure 11a.

Figure $11 \mathrm{~b}$ shows the dependence of lateral displacement $\delta$ with the time of the day. The azimuth angle of the Sun position varies by $180^{\circ}$ daily, but the acceptance angle of the system is limited to $45^{\circ}$; therefore, the working time of the system is limited to about $7 \mathrm{~h}$ per day (9:00 a.m.-4:00 p.m.). The movement amplitude of daily tracking of about $43 \mathrm{~mm}$ can be determined based on Figure 11b. Because the zenith angle of Sun position ranges from $-23.5^{\circ}$ to $23.5^{\circ}$ within each year, seasonal tracking (Sun tracking along north-south direction) requires the displacement range of $19 \mathrm{~mm}$. lateral displacement of Sun-tracking allows using step-motors that can be controlled by predetermined functions based on known equations of solar motion instead of using optical sensors and tracking algorithms as usual. The new Sun-tracking mechanism is expected to eliminate the tracking error and support mechanism against wind load and simplify the overall tracking infrastructure. 


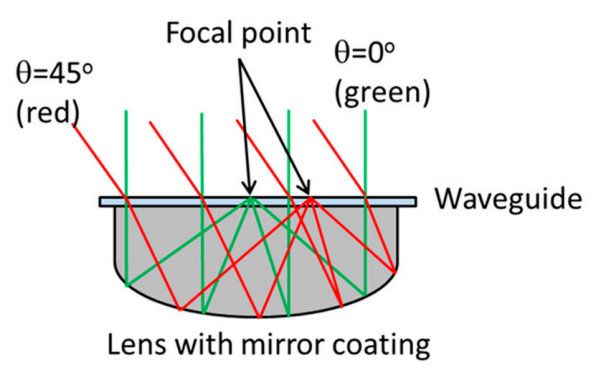

(a)

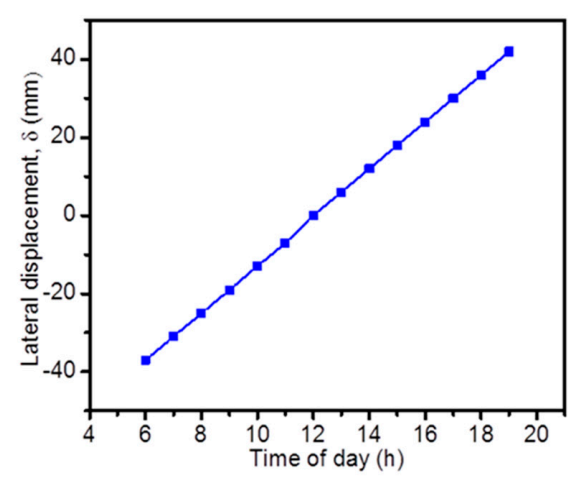

(b)

Figure 11. (a) Illustration of focal point displacement when sunlight incident angle changes from $0^{\circ}$ to $45^{\circ}$; (b) Dependence of displacement of focal point on the course of the day.

\subsection{Discussion}

Our goal is to illuminate a working plane of an indoor area with an illuminance of 500 lux, which is the minimum requirement for working space according to European standard [25]. At the highest elevation of the Sun at 12:30 p.m., the output flux is about 13,600 lm, as shown in Figure 10b. Daylighting systems with optical fibers are being used to deliver sunlight deep into a remote area of the building; therefore, the transmission loss of POF is the most important impact. Assume that the OFDS is installed on the roof of the building to illuminate a room at $6 \mathrm{~m}$ below from the top, about $10 \mathrm{~m}$ length of $\mathrm{POF}$ is required from the concentrator part to the illumination area. The transmission loss of $2 \mathrm{~mm}$ core diameter POF is $0.46 \mathrm{~dB} / \mathrm{m}$. This means that the luminous flux decreases by 2.8 times when propagating along the $10 \mathrm{~m}$ length of POF. Therefore, a luminous flux of $4850 \mathrm{~lm}$ can be delivered to the interior. The ratio between luminous flux of $4850 \mathrm{~lm}$ and the required illuminance of $500 \mathrm{lux}$ shows that the proposed system can illuminate for $\sim 10 \mathrm{~m}^{2}$. At other times of the day, the sunlight in the interior becomes less than the required value of 500 lux, and the artificial light sources should be turned on to compensate.

As results in Section 3.1, one of the significant losses (25\%) came from mismatching between the POF bundle and exit port of waveguide. It is fortunate, however, that flat POFs developed recently can provide a new approach. The design and fabrication method of flat POFs was mentioned in a US patent [26]. Some types of flat POFs were commercialized for lighting purposes. Figure 12a shows the commercial flat POFs with different sizes [24]. Because the cross-section of flat fiber is a rectangular shape, then it will perfectly match with the exit port of waveguide. Figure $12 \mathrm{~b}$ shows the configuration of flat POFs coupled with the planar waveguide. By using flat fiber, the mismatching loss can be reduced from $25 \%$ to less than $3 \%$. However, there is a trade-off between using cylindrical POF and flat POF. The attenuation of flat POF is higher than cylindrical POF. So if transport distant is long, using the flat POF is less effective. Depending on each specific circumstance, the use of conventional POF or flat POF will be decided.

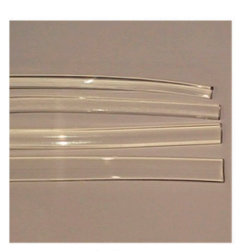

(a)

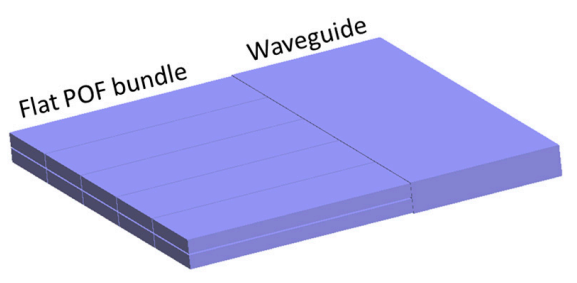

(b)

Figure 12. (a) Commercial flat POF with different sizes; (b) Alternative approach for optical fiber-planar waveguide coupling using a bundle of flat POFs. 
Any daylighting system is facing a major challenge with respect to cost-efficiency. The main purpose of our design is the development of a system that can allow the possibility of low fabrication cost. The waveguide, lens array and POFs of the daylighting system concept proposed here are made of a single material-PMMA that contribute to reducing manufacturing cost and recycling cost. From the viewpoint of fabrication, lens array has very simple structure so that it would be fabricated by injection molding process. As suggested by Karp et al. [23], the roll processing is possible for a planar waveguide with the low-cost material. All these features lead to a huge reduction in manufacturing cost. One disadvantage of our proposed system is lower concentration ratio in comparison with conventional systems, which results in a higher number of optical fibers for the same amount of light. Using a high number of optical fibers increases the system, but optical fiber cost has become much cheaper recently. On the other hand, to achieve high concentration ratio in the conventional system, very high quality of optical devices, precise alignment and Sun-tracking system are required. Our proposed system can overcome most of the challenges that contribute to increasing the system cost.

\section{Conclusions}

A flat OFDS using a planar waveguide has been proposed and analysed. The flat OFDS includes a mirror-coated lens array, a planar waveguide with redirecting prism. The concentrated sunlight beams from mirror-coated lens array are redirected and coupled with waveguide by a cone-shaped prism. Coupled sunlight is guided inside waveguide by TIR mechanism. The thickness of system of $35 \mathrm{~mm}$ was achieved while the length and width of the system were $500 \mathrm{~mm} \times 500 \mathrm{~mm}$. The simulation results indicate that $51.4 \%$ of optical efficiency was achieved at a concentration ratio of $C_{\text {geo }}=125$. One interesting aspect is that the system supports utilizing a lateral displacement Sun-tracking mechanism which can reduce the complexity and cumbersome work in comparison with the conventional Sun-tracking mechanism. The proposed flat OFDS is very suitable for daylighting purposes which mostly can be installed on a building's rooftop.

Acknowledgments: This work was supported by the National Research Foundation of Korea (NRF) grant funded by the Korea government (MSIP) (No. 2014R1A2A1A11051888); 2017 Research Fund of Myongji University.

Author Contributions: Ngoc Hai Vu conceived and developed the original ideas. Ngoc Hai Vu carried out the performance analysis and simulations, and wrote the paper. Seoyong Shin supervised the research and finalized the paper.

Conflicts of Interest: The authors declare no conflict of interest.

\section{References}

1. Vu, N.-H.; Shin, S. Optical fiber daylighting system combined with LED lighting and CPV based on stepped thickness waveguide for indoor lighting. J. Opt. Soc. Korea 2016, 20, 488-499. [CrossRef]

2. Vu, N.-H.; Pham, T.-T.; Shin, S. Modified optical fiber daylighting system with sunlight transportation in free space. Opt. Express 2016, 24, A1528-A1545. [CrossRef] [PubMed]

3. Krarti, M.; Erickson, P.M.; Hillman, T.C. A simplified method to estimate energy savings of artificial lighting use from daylighting. Build. Environ. 2005, 40, 747-754. [CrossRef]

4. Volotinen, T.T.; Lingfors, D.H.S. Benefits of glass fibers in solar fiber optic lighting systems. Appl. Opt. 2013, 52, 6685-6695. [CrossRef] [PubMed]

5. Mayhoub, M.S. Innovative daylighting systems' challenges: A critical study. Energy Build. 2014, 80, $394-405$. [CrossRef]

6. Sapia, C. Daylighting in buildings: Developments of sunlight addressing by optical fiber. Sol. Energy 2013, 89, 113-121. [CrossRef]

7. Lingfors, D.; Volotinen, T. Illumination performance and energy saving of a solar fiber optic lighting system. Opt. Express 2013, 21, A642. [CrossRef] [PubMed]

8. Tsuei, C.-H.; Sun, W.-S.; Kuo, C.-C. Hybrid sunlight/LED illumination and renewable solar energy saving concepts for indoor lighting. Opt. Express 2010, 18 (Suppl. 4), A640-A653. [CrossRef] [PubMed] 
9. PARANS Product Information. Available online: http://www.parans.com/eng/sp3/ (accessed on 14 December 2015).

10. HIMAWARI Solar Lighting System. Available online: http://www.himawari-net.co.jp/e_page-index01.html (accessed on 14 December 2015).

11. Vu, N.-H.; Shin, S. Cost-effective optical fiber daylighting system using modified compound parabolic concentrators. Sol. Energy 2016, 136, 145-152. [CrossRef]

12. Vu, N.; Shin, S. A Large Scale Daylighting System Based on a Stepped Thickness Waveguide. Energies 2016, 9, 71. [CrossRef]

13. Price, J.P.; Giebink, N. Concentrating photovoltaic panels for the rooftop. SPIE Newsroom 2015, 2, 2-4. [CrossRef]

14. Price, J.S.; Sheng, X.; Meulblok, B.M.; Rogers, J.A.; Giebink, N.C. Wide-angle planar microtracking for quasi-static microcell concentrating photovoltaics. Nat. Commun. 2015, 6, 6223. [CrossRef] [PubMed]

15. Dhakal, R.; Lee, J.; Kim, J. Bio-inspired thin and flat solar concentrator for efficient, wide acceptance angle light collection. Appl. Opt. 2014, 53, 306-315. [CrossRef] [PubMed]

16. Winston, R.; Gordon, J.M. Planar concentrators near the étendue limit. Opt. Lett. 2005, 30, $2617-2619$. [CrossRef] [PubMed]

17. Chien, M.-C.; Tung, Y.L.; Tien, C.-H. Ultracompact backlight-reversed concentration optics. Appl. Opt. 2009, 48, 4142-4148. [CrossRef] [PubMed]

18. Vu, N.-H.; Shin, S. Development of daylighting systems with non-imaging concentrator. Proc. SPIE 2015, 9572. [CrossRef]

19. Karp, J.H.; Ford, J.E. Planar micro-optic solar concentration using multiple imaging lenses into a common slab waveguide. Proc. SPIE 2009, 7407. [CrossRef]

20. Bouchard, S.; Thibault, S. GRIN planar waveguide concentrator used with a single axis tracker. Opt. Express 2014, 22, A248-A258. [CrossRef] [PubMed]

21. Selimoglu, O.; Turan, R. Exploration of the horizontally staggered light guides for high concentration CPV applications. Opt. Express 2012, 20, 19137-19147. [CrossRef] [PubMed]

22. Bouchard, S.; Thibault, S. Planar waveguide concentrator used with a seasonal tracker. Appl. Opt. 2012, 51, 6848. [CrossRef] [PubMed]

23. Karp, J.H.; Tremblay, E.J.; Ford, J.E. Planar micro-optic solar concentrator. Opt. Express 2010, 18, 1122-1133. [CrossRef] [PubMed]

24. Plastic Optical Fiber. Available online: http://www.hktdc.com/suppliers-products/Plastic-Optical-FiberCable/en/1X00K2VZ/2984370/ (accessed on 21 July 2017).

25. European Standard: Light and Lighting-Lighting of Work Places_Part 1: Indoor Work Places. Available online: https://www.en-standard.eu/csn-en-12464-1-light-and-lighting-lighting-of-workplaces-part-1-indoor-work-places / (accessed on 27 September 2017).

26. Cappellini, P.; Pierluigi, C.; Hassan, B. Flat Plastic Optical Fiber and Illumination Apparatus Using Such Fiber. U.S. Pantent No. 2004/0264899 A1, 30 December 2004.

(C) 2017 by the authors. Licensee MDPI, Basel, Switzerland. This article is an open access article distributed under the terms and conditions of the Creative Commons Attribution (CC BY) license (http://creativecommons.org/licenses/by/4.0/). 